\title{
Planar Drawings of Origami Polyhedra ${ }^{\star}$
}

\author{
Erik D. Demaine and Martin L. Demaine
}

Dept. of Computer Science, Univ. of Waterloo, Waterloo, Ontario N2L 3G1, Canada, \{eddemaine, mldemaine\}@uwaterloo.ca

This work studies the structure of origami bases via graph drawings of origami polyhedra. In particular, we propose a new class of polyhedra, called extreme-base polyhedra, that capture the essence of "extreme" origami bases. We develop a linear-time algorithm to find the "natural" straight-line planar drawing of these polyhedra. This algorithm demonstrates a recursive structure in the polyhedra that was not apparent before, and leads to interesting fractals.

Our hope is to discover fundamental properties of paper folding through graph drawing. One important problem in origami mathematics is to find a useful characterization of when a crease pattern can be folded flat (using every crease). This problem is known to be NP-hard [3]. However, this does not preclude the possibility of a simple characterization, only the tractability of such a characterization. For example, one conjecture that we have arrived at from this work is that every flat-foldable crease pattern has a Hamiltonian cycle. This property is insufficient, but a similar condition that is NP-hard to verify yet easy to understand may fill the gap in understanding flat origami.

Origami bases are the starting point for designing origami models. An extreme origami base maps the boundary of a polygonal piece of paper (convex for our purposes) to a common plane. Two examples of extreme bases are given in Fig. 1(c). The crease pattern of an extreme base is the union of the medial axis of the polygon and "perpendicular folds." One interesting property of these extreme bases is that they can be folded while keeping the faces rigid.

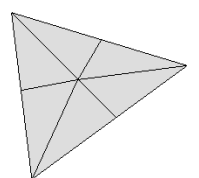

(a)

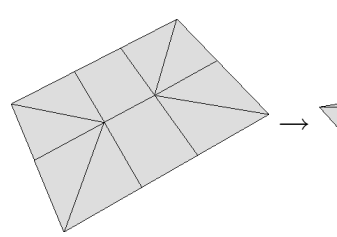

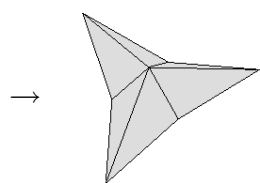

(b)

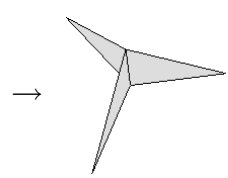

(c)

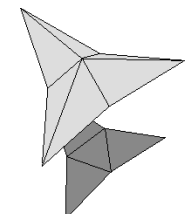

(d)

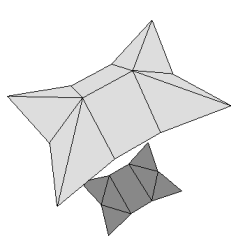

Fig. 1. (a-c) Folding of an extreme base for a triangle (top row) and quadrilateral (bottom row). (d) Polyhedra formed from partial foldings; the lower convex hull is drawn below.

\footnotetext{
* Supported by NSERC.
} 
In order to understand how the boundary of an extreme base becomes coplanar, we add the lower convex hull to a partially folded extreme base, thus forming an extreme-base polyhedron; see Fig. 1(d). It can be shown that the topology of this polyhedron is independent of the stage of the partial folding. This work examines planar drawings of these polyhedra. We consider this infinite class of polyhedra to be part of a more general class of origami polyhedra, which come from partial foldings of other origami bases.

A simple way to draw extreme-base polyhedra is to take the crease pattern and add curved edges representing the lower convex hull; see Fig. 2 (left). Basically, there is a cycle of curved edges connecting the ends of the perpendicular folds emanating from a common vertex of the medial axis.

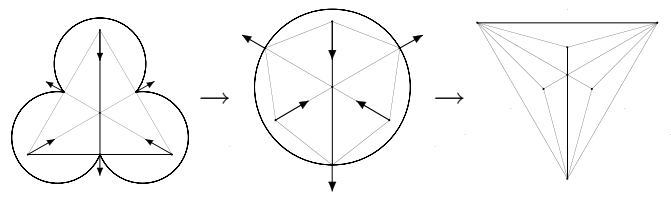

Fig. 2. Left: Curved drawing of the polyhedron from Fig. 1 Middle and right: "Stretching" into a drawing with straight lines.

One can imagine "stretching" the outside cycle to straighten out the curves, and recursing until we have a straight-line planar drawing; see Fig. 2 In the full version of this paper [1], we describe a linear-time algorithm for computing such a drawing directly from the topology of the medial axis, producing what we call the natural drawing; see Fig. 3.
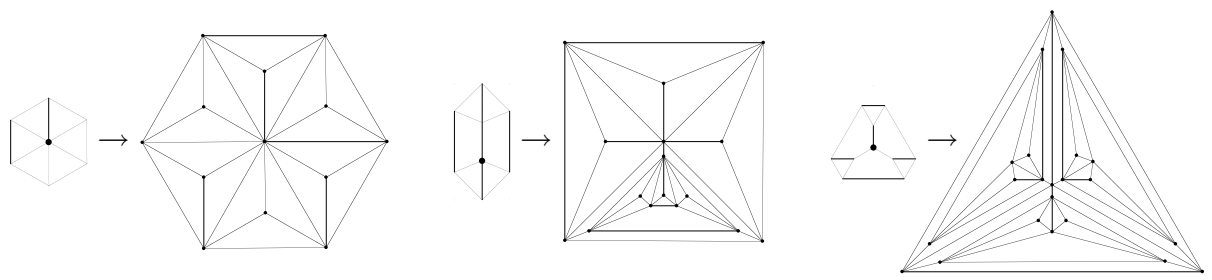

Fig. 3. The natural drawings of extreme-base polyhedra for hexagonal pieces of paper.

One advantage of the natural drawing is that it displays a recursive structure in the polyhedra. By lazily generating an infinite tree (in principle representing the medial axis of a polygon with an infinite number of vertices) the algorithm produces interesting fractals; examine Fig. 3 for an idea. To help view these fractals, as well as complex finite drawings, we introduce a zoom feature that is similar in spirit to fisheye views [2] but retains the geometry in order to display the recursive structure. 


\section{References}

1. E. D. Demaine and M. L. Demaine. Planar drawings of origami polyhedra. Technical Report CS-98-17, University of Waterloo, August 1998.

2. E. G. Noik. A survey of presentation emphasis techniques for visualizing graphs. In Proc. Graphics Interface, Banff, Canada, May 1994, 225-233.

3. M. Bern and B. Hayes. The complexity of flat origami. In SODA, Atlanta, Jan. 1996, $175-183$. 